\title{
EpiCore: Crowdsourcing Health Professionals to Verify Disease Outbreaks
}

\author{
Jennifer M. Olsen* \\ Skoll Global Threats Fund, San Francisco, CA, USA
}

\section{Introduction}

EpiCore draws on the knowledge of a global community of human, animal, and environmental health professionals to verify information on disease outbreaks in their geographic regions. By using innovative surveillance techniques and crowdsourcing these experts, EpiCore enables faster global outbreak detection, verification, and reporting.

\section{Methods}

Through a secure online platform, members are able to easily and quickly provide local information to expedite outbreak verification. EpiCore volunteer applications are vetted to ensure that they possess the public health and epidemiologic expertise necessary to contribute to the platform.

\section{Results}

EpiCore currently has over 1600 members that span 135 countries. During the first 8 months of EpiCore's launch, 172 requests for information to volunteers have been posted with an average response rate of over $80 \%$.

\section{Conclusions}

With its geographical distribution of members and high response rate, EpiCore is poised to enable the world to verify potential outbreak signals faster. By improving situational awareness, de-escalating rumors or false information, and corroborating using other existing sources, EpiCore is able to reduce the signal to noise ratio in disease surveillance. Hence, by detecting and verifying outbreaks faster, health officials can generate early responses that can curb epidemics and save lives.

\section{Keywords}

Disease Verification; crowdsourcing; online platform

\section{Acknowledgments}

EpiCore was initiated as a partnership between the Skoll Global Threats Fund, HealthMAP, ProMED, and TEPHINET.

\section{*Jennifer M. Olsen}

E-mail: jolsen@skollglobalthreats.org 